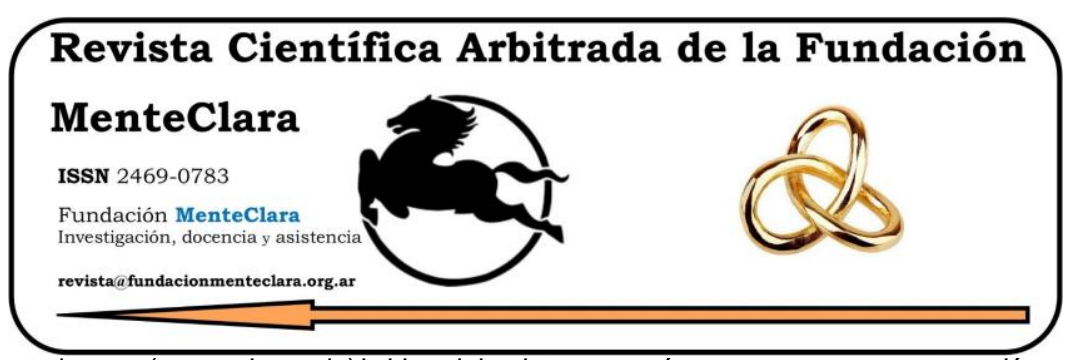

Artículos atravesados por (o cuestionando) la idea del sujeto -y su género- como una construcción psicobiológica de la cultura. Articles driven by (or questioning) the idea of the subject -and their gender- as a cultural psychobiological construction.

Vol. 6 (2021), enero-diciembre ISSN 2469-0783

https: / / datahub.io/ dataset/2021-6-e251

\title{
DOBLE PRESENCIA, ENTRE LA FAMILIA Y EL TRABAJO
}

\author{
DOUBLE PRESENCE, BETWEEN FAMILY AND WORK
}

Sandra Sorbara ${ }^{1}$, Silvana Baró2 ${ }^{2}$ Rocío Greco ${ }^{3}$, Mirian Preiti ${ }^{4}$ y Myriam Quinteros ${ }^{5}$

Cómo citar este artículo / Citation: Sorbara, S; Baró, S; Greco, R; Preiti, M y Quinteros, M. (2021). Doble presencia, entre la familia y el trabajo. Revista Cientifica Arbitrada de la Fundación MenteClara, Vol. 6 (251). DOI: https://doi.org/10.32351/rca.v6.251

Copyright: (C) 2021 RCAFMC. Este artículo de acceso abierto es distribuido bajo los términos de la licencia Creative Commons Attribution 4.0 International License (CC BY 4.0). Recibido: 27/08/2021. Aceptado: 14/09/2021 Publicación online: 16/09/2021

Conflicto de intereses: Ninguno que declarar.

Fuente de financiación: Secretaría de Investigación y de sarrollo de la Universidad de Flores.

\section{Resumen}

La investigación indagó la prevalencia de exposición de los factores de riesgo psicosociales en los docentes de nivel medio. Se realizó un estudio descriptivo transversal de diseño cuantitativo. El objetivo de este artículo es visualizar la importancia del trabajo de los docentes que se enfrenta en tiempo de pandemia una

\footnotetext{
${ }^{1}$ Universidad de Flores. Facultad de Psicología y Ciencias Sociales, Argentina. sandrasor1212@gmail.com

2 Universidad de Flores. Facultad de Psicología y Ciencias Sociales, Argentina. silvana_baro@yahoo.com.ar
}

${ }^{3}$ Universidad de Flores. Facultad de Psicología y Ciencias Sociales, Argentina. grecorocio326@gmail.com

\footnotetext{
${ }^{4}$ Universidad de Flores. Facultad de Psicología y Ciencias Sociales, Argentina. mirianpreiti@hotmail.com

5Universidad de Flores. Facultad de Psicología y Ciencias Sociales, Argentina. myriam835@hotmail.com
} 
doble demanda. Como instrumento para la recolección de datos se utilizó el cuestionario CoPsoQ-ARG validado para la población argentina, con el fin de establecer los niveles de exposición. Los hallazgos demostraron un nivel de exposición alto en el grupo de mujeres $(77,3 \%)$ a diferencia del grupo de hombres $(22,7 \%)$.

\begin{abstract}
There search investigated the prevalence of exposure to psychosocial risk factors in high school teachers. A descriptive, cross-sectional stud y with a quantitative design was carried out. The objective of this article is to visualize the importance of the work of teachers that faces a double demand in times of a pandemic. As an instrument for data collection, the CoPsoQ-ARG question air revalidated for the Argentine population was used, in order to establish exposure levels. The findings demonstrated a high level of exposure in the group of women (77.3\%) compared to the group of men $(22.7 \%)$.
\end{abstract}

Palabras Claves: Factores de riesgo psicosociales; docentes de nivel medio; doble presencia

Keyw ords: Psycho social risk factors; inter mediate level teachers; double presence 


\section{Introducción}

A partir de la investigación prevalencia de exposición a factores de riesgo psicosociales en docentes de escuelas medias de CABA, se indagó sobre la relación entre el trabajo asalariado y el trabajo domésticofamiliar en contexto de pandemia. Cabe aclarar que el objetivo de esta investigación fue evaluar los factores de riesgo psicosociales en el trabajo entre los docentes de escuelas de nivel medio del ámbito privado de CABA.

La relación entre el trabajo y la salud ha estado presente desde tiempos inmemorables, al ser el trabajo una actividad sustantiva de los seres humanos. Por tal motivo el trabajo y la salud son procesos complejos y relacionados entre sí (Roca et al., 2011) (Tomasina, 2012). Ambos dependen de la interacción de múltiples factores sociales, políticos, económicos, culturales y científicos (Alcántara Moreno, 2008), por lo tanto son considerados fenómenos sociales que interactúan y se combinan en un alto grado de complejidad como ocurre con los hechos humanos donde entra en acción una elevada cantidad de variables (Alcántara Moreno, 2008) (Tomasina, 2012).

Cabe mencionar también que las condiciones fueron cambiando y mejorando, horas trabajadas, ruidos, ambientes e higiene. Tales efectos repercutieron de manera positiva disminuyendo la morbilidad y la siniestralidad en el trabajo. El número de enfermedades laborales y de accidentes se ha controlado parcialmente y ha disminuido su incidencia. Pero, probablemente, la mayor modificación se ha producido en el cambio del concepto de salud laboral que ha dejado de ser un problema individual para convertirse en un problema social y empresarial y principalmente, en un derecho del trabajador (Ley No 24.430, 1995). 
Desde hace varios años la atención de la salud del trabajador estuvo centrada en riesgos físicos, químicos y ambientales (Moreno Jiménez \& Báez León, 2011). La importancia de centrarse en estos riesgos radica en que tales factores son causantes de la gran mayoría de los accidentes y enfermedades laborales (Moreno-Jiménez, 2014) (Moreno Jiménez \& Báez León, 2011).

Los factores de riesgos laborales vienen constituyendo una preocupación hace varias décadas, ya en los años 70 toma repercusión (Moreno-Jiménez \& Báez Leon, 2010). Actualmente, se diferencian tres formas de referirse a los aspectos psicosociales, los factores psicosociales en el trabajo, los factores psicosociales de riesgo y los riesgos laborales (Castillo, Santana, Valeta, Alvis, \& Romero, 2011) (Demaria et al., 2017; Moreno-Jiménez, 2011).

Los factores psicosociales laborales están íntimamente relacionados con la cultura organizacional, condiciones de trabajo, factores interpersonales, condiciones ergonómicas, condiciones salariales, condiciones personales o individuales (Villalobo, 1997) (Gil-Monte 2012), tienen capacidad para afectar tanto al bienestar como a la salud fisica, psíquica o social del trabajador, como también al desarrollo del trabajo (Kalimo, El-Batawi \& Cooper, 1998) (Moreno-Jiménez \& Báez Leon, 2010) (Feldman\& Blanco, 2012).

Dentro del colectivo de trabajo los factores de riesgo repercuten mayormente en poblaciones que se encuentran trabajando en contacto con otras personas diariamente, médicos, enfermeros y docentes. No solo atienden problemáticas de índole académicas, también se involucran con problemáticas individuales de los alumnos. 
Por tal motivo los factores psicosociales dependen de la dirección que tomen, podrán influir de manera positiva o negativa sobre la salud del docente. Si es positiva, repercute en una adecuada salud laboral, generando en el docente un alto desempeño y otorgándole satisfacción en su actividad siendoun medio para que las personas puedan realizarse, dar sentido a sus vidas y ser creativas. Por otro lado, el trabajo puede proporcionar estatus y prestigio social, como también constituir una fuente importantísima de identidad personal y social, porque el modo en cómo la persona se ve a sí misma tiene relación con cómo percibe que es vista por los demás. Indudablemente el trabajo tiene importantes implicancias de carácter económico ya que permite sobrevivir a los individuos y conseguir bienes de consumo. Al mismo tiempo, puede ser una fuente de oportunidades para la interacción y los contactos sociales (Andrade Jaramillo, 2014) (OIT, 2005).

Si por el contario, influye de manera negativa genera múltiples efectos en la salud física y mental del docente; problemas mentales, cardiovasculares u osteomusculares, convirtiéndose en un riesgo potencial para la salud (Mansilla Izquierdo, 2014) (Moreno-Jiménez, 2014)

Desde finales del 2019 el mundo se vio convulsionado con una nueva amenaza para la humanidad. La pandemia de COVID-19 afectó a la población mundial, produciendo cambios especialmente en el mundo del trabajo. El 2 de marzo de 2020 se detecta en Argentina el primer caso de COVID 19 positivo, ese mismo mes el Gobierno Nacional emite el Decreto 297/2020 manifestando el Aislamiento Social Preventivo y Obligatorio por el término de un año (Boletín Oficial de la República Argentina, 2020). Las condiciones laborales se vieron afectadas, las escuelas cerraron sus puertas y los docentes comenzaron a trabajar desde sus casas, adaptándose a nuevas situaciones, con nuevos materiales y 
herramientas tecnológicas. El nuevo aprendizaje impuesto, el trabajo realizado dentro del hogar, donde el docente se encuentra realizando tareas laborales junto a su familia y la responsabilidad que existe entre sostener el trabajo y la tareas domésticas-familiares genera desgaste, preocupación por no llegar a concluir tareas, malestar, insatisfacción y desgano (Serrano et al., 2020). La intensidad del tiempo invertido y la exigencia han aumentado impactando considerablemente la vida y la salud de los individuos. La virtualidad se encuentra sujeta más al tiempo que al espacio geográfico, borrando las fronteras entre el tiempo laboral y el familiar.

Asimismo, el área docente se encuentra conformado mayoritariamente por mujeres, y son ellas quienes en gran parte se responsabilizan del trabajo doméstico, las tareas de cuidado y asistencia en las tareas escolares de los hijos, ello implica que la carga de trabajo se multiplique en medio del contexto de aislamiento, lo que conlleva a problemáticas de salud y a una mayor predisposición del cuerpo atravesado por múltiples demandas (Behcer, 2020).

Estas situaciones generan patologías fisicas, contracturas musculares y dolores de cabeza, malas posturas, repetición de movimientos, mantenimiento de una posición estática de forma prolongada en el tiempo, y sedentarismo. Asimismo, la sobre demanda en el trabajo, el trabajo a destiempo, falta o poco de tiempo de esparcimiento, aislamiento de los vínculos sociales entre otros generan y desorganización en el tiempo/espacio generan consecuencias emocionales que impactan en la salud mental, angustia, ansiedad, tensión, molestia e irritabilidad (Behcer, 2020). 


\section{Materiales y Métodos}

La investigación se desarrolló a partir de un estudio cuantitativo, descriptivo de corte transversal, que permitió describir el fenómeno estudiado. El muestreo no probabilístico por conveniencia del investigador, participaron docentes de nivel medio de instituciones de gestión privada del ámbito de CABA. Las personas se encontraron en sus puestos de trabajo

Como instrumento de recolección de datos se utilizó el COPOSQ ARG GRUP, el mismo permite evaluar los factores de riesgos psicosociales y medir aquellas características de la organización que pueden representar un riesgo para la salud el bienestar de los trabajadores/as (Zelaschi, et al, 2021). Consta de 7 dimensiones, y en cuyo cuestionario se incluye una preguntas con cinco opciones de respuesta: siempre; la mayoría de las veces; algunas veces; solo unas pocas veces y nunca. La puntuación del cuestionario está dada por una escala Likert, que se asocia a cada pregunta, con un máximo de 4 puntos por pregunta, donde una mayor puntuación indica un mayor riesgo. A partir de ello, se estimaron los puntajes de cada dimensión con el objeto de establecer rangos: Bajo (nivel de exposición más favorable para la salud), Medio (nivelintermedio) y Alto (nivel de exposición más desfavorable para la salud).

\section{Resultados}

En el marco de la investigación cuantitativa de Baró et al (2021) se estudió la doble presencia entre el grupo de trabajadores del ámbito docente de instituciones educativas de gestión privada. La investigación, cuyo objetivo principal era evaluar los factores de riesgo psicosociales en el trabajo entre los docentes de escuelas de nivel medio del ámbito 
privado de CABA, consideró entre sus dimensiones de análisis la doble presencia y la diferencia entre hombres y mujeres.

Respecto de la dimensión estudiada se observó que existen diferencias significativas entre hombres y mujeres en momento de realizar las tareas relacionadas al trabajo remunerado y el trabajo doméstico-familiar, los datos obtenidos muestran puntuaciones elevadamente altas el grupo de mujeres en el nivel alto 77,3\% (N=198), a diferencia del grupo de hombres donde el porcentaje más alto de exposición se observa en el nivel bajo 42,9\% ( $\mathrm{N}=198)$. Cabe mencionar que estas puntuaciones implican que los hombres perciben mayor apoyo social y estima, de esta manera sostiene una mejor salud. A diferencia de las mujeres donde recae la necesidad de responder a las demandas del espacio de trabajo domésticofamiliar y a las demandas del trabajo asalariado. Equivale a una presión doble para responder a ambas demandas cuando se producen de manera simultánea, así se observa en la Tabla 1:

Tabla 1: Elaboración propia en base a los datos obtenidos

\begin{tabular}{|l|l|l|}
\hline Nivel de exposición & Hombre & Mujer \\
\hline Bajo & $42,9 \%$ & $57,1 \%$ \\
\hline Medio & $31,7 \%$ & $68,3 \%$ \\
\hline Alto & $22,7 \%$ & $77,3 \%$ \\
\hline
\end{tabular}

Fuente: elaboración propia sobre la muestra obtenida. Baró et al., (2021).

\section{Discusión}

Los resultados obtenidos están en concordancia con diferentes autores. La doble presencia responde históricamente al constructo patriarcal que ha acompañado a las mujeres desde el inicio de la 
humanidad, que le asigna la función del cuidado y la atención de las necesidades básicas de los demás. Por esto, el papel de la mujer fuera del lugar de trabajo se extiende al ámbito doméstico, con funciones de gestión del cuidado con sus hijos, la casa y su pareja. En el ámbito laboral, este factor incrementa las cargas fisicas y psíquicas que pueden alterar la salud, deteriorar las relaciones con los colegas y aumentar la posibilidad de errores en el trabajo. En el ámbito personal, la doble presencia produce dificultades extra laborales, como la disminución del contacto social y familiar, lo cual afecta la vida en parejay la organización de tiempos y horarios. Esto impacta la vida familiar y genera estrés laboral. También se afecta la vida social, las redes de apoyo, el esparcimiento, la utilización del tiempo libre y las posibilidades de cuidado de la salud (Vázquez, Ramírez \& Bello 2019).

Asimismo, a nivel físico la persona puede sufrir casos de estrés, ansiedad, dolores musculares, enfermedades cardiovasculares, etc. (GilMonte, 2012); a nivel psíquico, la carga mental, etc.; en su ámbito familiar, conflictos conyugales, incomodidad por el ruido de los niños, etc.; y, en la organización, falta de interés por el trabajo, ausentismo laboral, discordia con sus compañeros de trabajo, etc. Algo a tener en cuenta de suma importancia a tener en cuenta es que no todas las mujeres tienen la misma resistencia a los factores de riesgo psicosocial, hay que considerar que la personalidad de toda mujer no viene acompañada por un patrón concreto, sino que se ve influenciada por factores como el nivel de aspiración de la persona, la confianza en sí mismo, la resistencia al estrés, personas con estas habilidades suelen ser más eficientes, responsables y activas.

El hecho de que la doble presencia sea un riesgo psicosocial habitual en las mujeres se debe a que tradicionalmente han sido quienes han organizado y realizado la mayor parte del trabajo doméstico familiar, 
incluso cuando la familia se mantiene únicamente a través del sueldo de la mujer es ella quien sigue realizando las tareas domésticas en mayor medida, que el estado de salud de las mujeres es peor cuanto mayor sea el número de personas en el núcleo familiar (Estevan Reina et al., 2014).

La doble presencia, genera desigualdad laboral entre hombres y mujeres, ya que las obligaciones domésticas impiden que las mujeres puedan dedicarse más específicamente a su puesto de trabajo o profesión, algo a lo que sí pueden acceder los hombres al no tener el peso del trabajo doméstico. De esta manera se limita el acceso de las mujeres a puestos de dirección, el estatus laboral de las mujeres disminuye a medida que aumentan sus obligaciones sociales. (Moreno-Jiménez \& Garrosa, 2013).

\section{Conclusión}

La investigación deja en evidencia la situación a la cual se enfrentan las mujeres que desarrollan su trabajo en el ámbito de la docencia. En su mayoría perciben bajo control en el desarrollo de la actividad altas exigencias psicológicas, poco apoyo de sus vínculos sociales, falta de vínculos que permitan el trabajo colaborativo y escasas recompensas. La necesidad de dar respuesta de manera simultánea a las demandas laborales y domesticas-familiares puede afectar de manera negativa a la salud. Tanto mujeres como hombres son vulnerables a dichas presiones solo que, sin duda, son las mujeres quienes presentan mayor vulnerabilidad a estas situaciones. 


\section{Referencias}

Alcántara Moreno, G. (2008). La definición de salud de la Organización Mundial de la Salud y la interdisciplinariedad. Sapiens, 9(1). Disponible en:

https:/ / biblat.unam.mx/es/revista/sapiens/articulo/la-definicion-de-salud-de-laorganizacion-mundial-de-la-salud-y-la-interdisciplinariedad-gustavo-alcantara-moreno

Andrade Jaramillo, V, \& Gómez, I. (2008). Salud Laboral En Colombia. Pensamiento Psicológico, 4(10), 9-25. Disponible en: https://dialnet.unirioja.es/ejemplar/206720

Andrade Jaramillo, V. (2014). Cambios en las relaciones de trabajo : paradojas y consecuencias en el actual mundo del trabajo. Revista Diversas Perspectivas en Psicología, 10(2), 337-351. Disponible en: http://www.scielo.org.co/pdf/dpp/v10n2/v10n2a11.pdf

Baró, S; Sorbara, S; Milazzo, J; Quinteros, M; Greco, R; Preiti , M; Faba , C Núñez, K \& Cejudo, C (2021). Prevalencia de exposición a Factores de Riesgo Psicosociales en Docentes. [No publicado] Universidad de Flores

Becher P. (2020). Educación en tiempos de pandemia. Condiciones laborales y percepciones sobre el trabajo docente virtual en la Ciudad de Bahía Blanca (Argentina). Revista Científica Educación, 4 (8). Disponible en: https://ri.conicet.gov.ar/handle/11336/122867

Boletín oficial de la República Argentina (2020). Decreto 297/2020 Aislamiento social preventivo y obligatorio. Ciudad de Buenos Aires, 19/03/2020. Disponible en:

https://www.boletinoficial.gob.ar/detalleAviso/primera/227042/20200320

Castillo, I. Y; Santana, M; Valeta, A; Alvis, L. R \& Romero, E. (2011). Factores de riesgo psicosociales del trabajo en médicos de una Empresa Social del Estado en Cartagena de Indias. Revista. Facultad Nacional de Salud Pública, 29 (4), 363-371. Disponible en : https://www.re dalyc.org/pdf/120/12021522002.pdf

Constitución de la Nación Argentina. Ley No 24.430, Honorable Congreso de la Nación Argentina. Fecha de sanción 14 de diciembre de 1994. Disponible en:

https://www.argentina.gob.ar/normativa/nacional/ley-24430-804

Demaria, M; Farías, M \& Acevedo, G (2017). Factores de riesgos psicosociales presentes en el ambiente de trabajo de enfermería en hospitales públicos de Córdoba, Jujuy, La Pampa y Santiago del Estero, Argentina. Revista de Salud Pública, 60-72. Disponible en:

https://revistas.unc.edu.ar/index.php/RSD/article/view/16790

Estevan Reina, L., Rodríguez Sánchez, R. M., Romero González, B., Rodríguez López, Á., \& Romo Sola, M. (2014). Doble presencia: un riesgo psicosocial que evidencia la desigualdad entre hombres y mujeres en la conciliación de la vida familiar y laboral. Rei docrea, 3: 172-179. Disponible en: https://digibug.ugr.es/handle/10481/32323

Gil-Monte, P. (2012). Riesgos psicosociale s en el trabajo y salud ocupacional. Revista Peruana de Medicina Experimental y Salud Pública, 29 (2). Disponible en:

http://www.scielo.org.pe/scielo.php?pid=S1726-463420120002000128tscript=sci_abstract

Kalimo, R, El-Batawi, M \& Cooper, C (1988). Los factores psicosociales en el trabajo y su relación con la salud. Disponible en http:/ /apps.who.int/iris/bitstream/handle/10665/37881/9243561022_spa.pdf;jsessionid= C706FA112C6DCAB41C4AF72686027774?sequence $=1$

Mansilla Izquierdo, F. (2014). Manual de Riesgos Psicosociales en el trabajo : Teoría y Práctica. Disponible en: https://es.slideshare.net/FranCedeo/manual-de-riesgos-psicosociales-en-eltrabajo 
Moreno Jiménez, B \& Báez León, C. (2011). Factores y riesgos psicosociales, formas, con secuencias, medidas y buenas prácticas. Instituto Nacional de Seguridad e Higiene en el Trabajo. Madrid. Disponible en: http://doi.org/10.1016/0277-9536(85)90255-2

Moreno-Jiménez, B. (2014). Los riesgos laborales psicosociales : marco conceptual y contexto socio económico. ORP Journal, 1, 4-18 Disponible en: https://www.orpjournal.com/index.php/ORPjournal/article/view/7

Moreno-Jiménez, B. y Garrosa, E. (2013). Salud Laboral. Riesgos laborales psicosociales y bienestar laboral. Madrid: Pirámide

OIT (2005). Trabajo decente - Trabajo seguro. In Congreso Mundial sobre Salud y Seguridad en el Trabajo. Disponible en: https://www.ilo.org/global/about-the-ilo/mission-andobjectives/features/WCMS_075279/lang--es/index.htm

Ramos, E. (2008). Doble presencia: salud y prevención. Clave Sindical, 5(1), 16-18. Disponible en: https://www.um.es/siegum/publicaciones/autores/documentos/Doble-presencia.pdf

Roca, R, Iglesias, L, Salgado, L, Suárez, O, Tomasina, F., Stolovas, N, Alonso, V (2011). Manual Básico en Salud, Seguridad y Medio Ambiente de Trabajo. Uruguay: Comisión permanente de procesos y condiciones de estudio, trabajo y medio ambiente laboral de la Universidad de la Republica. Disponible en: http:/ / www.higiene.edu.uy/secdir/assets/manual3.pdf

Superintendencia de riesgos del trabajo (2015). Estudio de adaptación y validación para Argentina del Cuestionario Psicosocial de Copenhague (CoPsoQ-ISTAS21). Disponible en: http:/ / biblioteca.srt.gob.ar/pergamo/documento.ph p?ui=38re cno=6149 \&id=SRT.3.6149

Tomasina, F. (2012). Los problemas en el mundo del trabajo y su impacto en salud. Revista de Salud Pública, 14(1), 56-67. Disponible en: https://scielosp.org/pdf/rsap/2012.v14suppl1/56$\underline{67 / \text { es }}$

Vásquez, M. M. O., Ramírez, Y. C. Z., \& Bello, G. P. (2019). Factores de riesgo psicosocial que afectan a los profesionales en enfermería. Revista Colombiana de Enfermería, 18(1), 5. Disponible en:

https://revistacolombianadeenfermeria.unbosque.edu.co/article/view/2308

Villalobos, G. (2004). Vigilancia Epidemiológica de los Factores Psicosociales. Aproximación Conce ptual y Valorativa. Ciencia \& Trabajo, 6, 197-201. Disponible en:

https://pesquisa.bvsalud.org/ portal/resource/es/li1-420807

Zelaschi, M. C., Cornelio, C., Reif, L., \& Amable, M. (2021). Validación de un cuestionario de ries gos psicosociales en población trabajadora argentina (COPSOQII-ISTAS21) Adaptación cultural y validación para la Argentina. Revista De Psicologia, 084. Disponible en:

https://doi.org/10.24215/2422572Xe084 